\title{
Use of a tissue adhesive to repair fetal bodies after dissection
}

\author{
G S Gau, K Napier, J Bhundia
}

\begin{abstract}
The repair of fetal bodies after dissection is extremely difficult because of the delicacy of the skin. A tissue adhesive, Histoacryl blue, was used to repair bodies that had been dissected either before or after immersion in formalin. The repairs were achieved rapidly and neatly. The technique was easy to learn and, providing the method of repair described was followed, there was no breakdown subsequently.

The body cavities and reflected skin surfaces were lightly dried with absorbent paper towels and the body loosely packed with cotton wool. "Holding lines" of glue were made and a mid-line strip of glue was applied to the sternum, abdomen, and skull vault, allowing the skin edges to align neatly. Care must be taken to avoid using excess glue as this produces an exothermic reaction.

It is recommended that if a fetus has to be repaired after dissection Histoacryl blue will give a good result.
\end{abstract}

Although the law and, to a lesser extent society, distinguish between the loss of a fetus before and after 28 weeks' gestation, mothers do not. Support groups such as the Stillbirth and Neonatal Death Society (SANDS) (28 Portland Place, London W1 4DE) and the media (The Lost Babies, That's Life, BBC, 9 November 1983) have recently caused the medical profession to realise that greater importance must be given to the management of perinatal death. Subsequently, the reformation of the 1962 Abortion Act in 1967 and 1990 , the ability of neonatologists to save lives earlier on in gestation, and the work of the Miscarriage Association (PO Box 24, Ossett, West Yorkshire WF5 9XG), mean that parental wishes over the care of their aborted fetus's body are becoming an issue in all pathology laboratories. This is evidenced by the increasing numbers of requests for disposal by burial. This means the laboratory should present the body as attractively as possible.

Repair of babies' bodies is generally performed by a continuous herringbone suture, but in the pre- 25 week gestation fetus and in those bodies that have been stored in formalin stitches tend to tear through the fragile skin and are therefore inadequate and ugly. In an attempt to find a more acceptable method of repair a tissue adhesive, Histoacryl blue (Braun, Melsungen, Germany), was used on a series of fetuses on whom post mortem examinations had been performed.

\section{Methods}

Fifteen fetuses with gestational ages ranging from 12-22 weeks were obtained. Five were immersed in formalin before and immediately after post mortem examination; five were dissected fresh then immersed in formalin before repair; and five were dissected fresh and immersed in formalin after repair.

Post mortem examination of thoracic and abdominal organs was performed with standard skin reflection from a longitudinal mid-line incision from the root of the neck to the umbilicus where it divided and continued to each mid-inguinal point. The rib cartilages were cut on each side and the sternum and anterior thoracic cage removed. To examine the brain skin was reflected from a mid-line incision from the bregma to the mid-scapular point. The skull cap was removed by a circumferential cut. All organs were removed for examination and none replaced subsequently.

For repair the body cavities and the reflected skin surfaces were lightly dried with absorbent paper towels. The body was then loosely packed with cotton wool. To hold the anterior thoracic cage in place a dab of adhesive was applied to the cotton wool before emplacement, and when secure, the adhesive was applied to the rib ends and the rib cage correctly reformed. "Holding lines" of glue were placed as shown (fig 1). A mid-line strip of glue on to the sternum and across the cotton wool in the abdomen allowed the skin edges to align neatly.

The skull was packed with cotton wool and a dab of adhesive allowed the skull cap to be stabilised. The reflected skin was then drawn across, using similar holding lines of adhesive as in the thorax and abdomen, and finally a mid-line strip was applied to the vault of skull permitting close apposition of the skin edges (fig 2).

\section{Results}

Those fetuses which had been dissected after immersion in formalin were repaired with great ease as the tissues were fixed in the predissected shape. Those that had been immersed in formalin after dissection were more difficult to manage due to fixation of the loosened skin in unnatural shapes. Those that were repaired before fixation were simple to manage because of the flexibility of the skin (figs 3 and 4).

In all cases the glue secured the skin edges in good apposition and they remained so for at least six weeks until the bodies were disposed of according to parental wishes. In one case, 


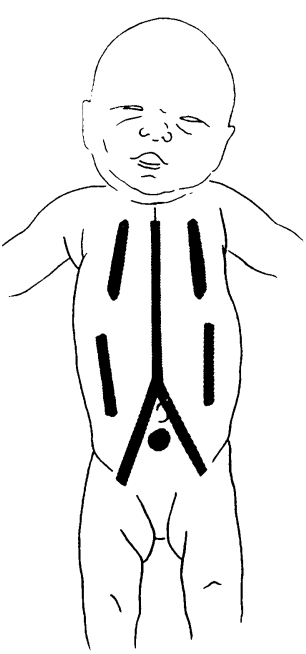

Figure 1 Diagram of a fetus to show the line of incision (\$): shaded areas indicate sites of application of Histoacryl blue.

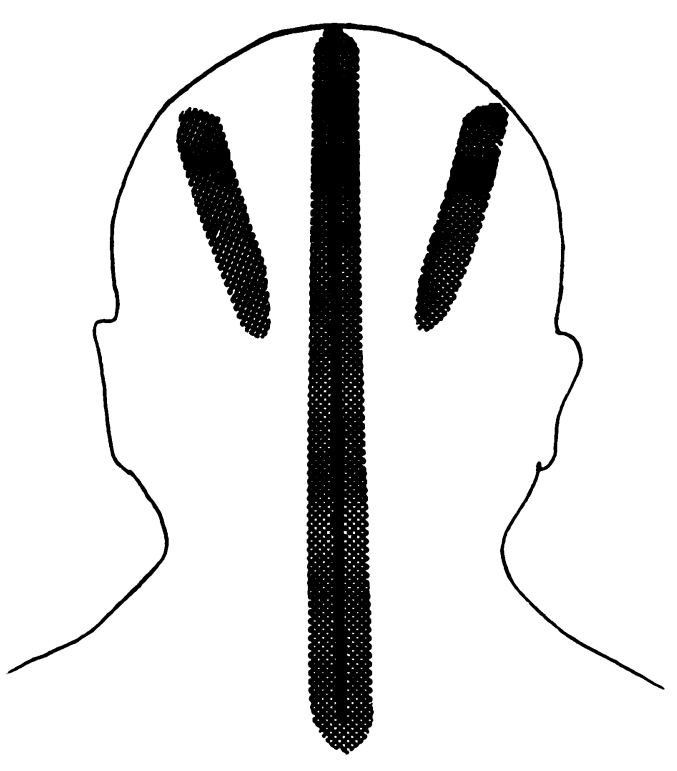

Figure 2 Diagram of the posterior aspect of a fetal skull. Incision and glue lines are indicated as in fig 1 .

which had been glued after fresh dissection where no holding lines had been used, the skin on either side of the glue joint tore away but the glued area remained fast.

\section{Discussion}

Suturing fetal bodies is extremely difficult, if not impossible, and time consuming. The fragile skin tears making it difficult to appose

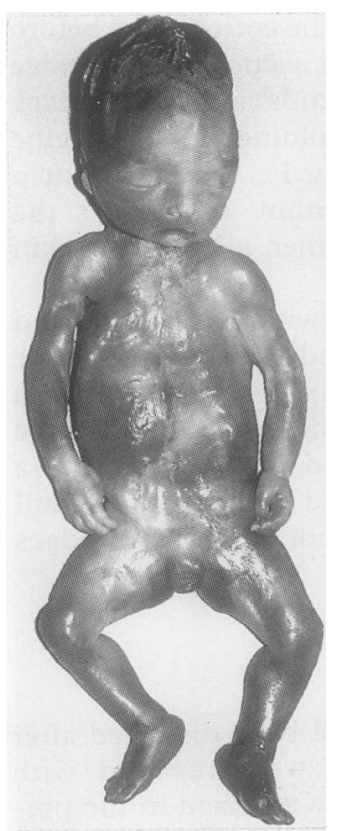

Figure 3 Twenty one week old fetus terminated for prolonged rupture of membranes, arrested growth, and sepsis, dissected and repaired in the fetus fresh state.

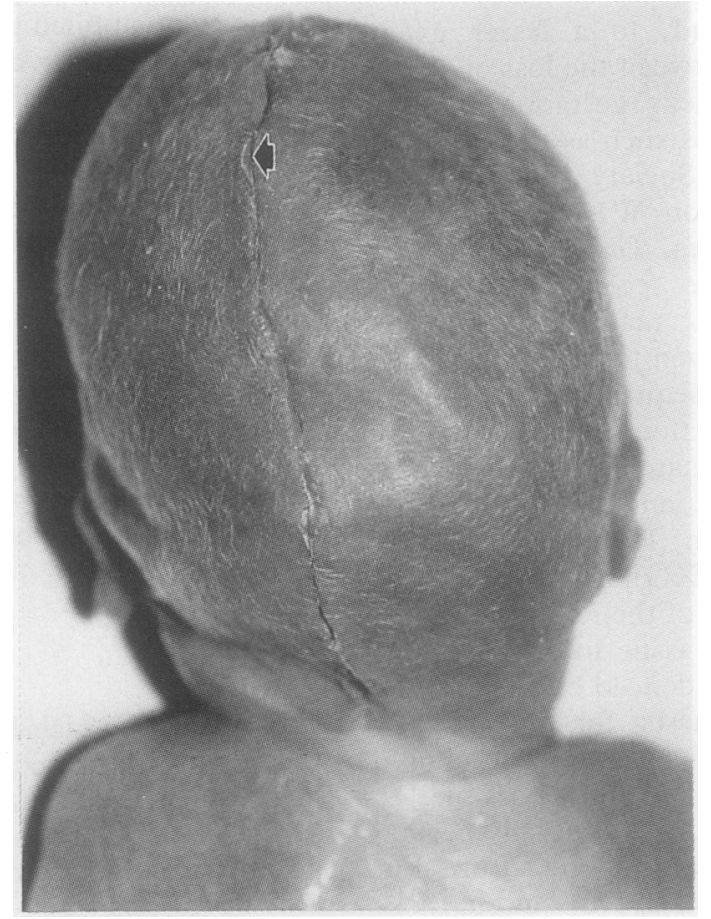

Figure 4 The repaired head of 23 week old fetus spontaneously aborted with intrauterine pneumonia and Some exothermic reaction can be seen. chorioamnionitis, dissected and repaired in the fresh state. skin edges accurately and to avoid puckering. If a fetal body has been immersed in formalin for even a short time the skin texture is such that suturing is impossible. Some neatness of repair can be obtained by using adhesive dressings but these are unattractive and cumbersome.

In this study Histoacryl blue, a cyano acrylate which polymerises on contact with the skin, was used. It is already widely used in surgical and dental practice. In this and postmortem work it undoubtedly gives a neat result which lasts long enough for the purpose intended. The technique of avoiding adherence of gloves and instruments is rapidly learnt, as is the technique of repairing the body itself. The repair procedure is extremely quick taking between 10-20 minutes for each body.

The major disadvantage found in our laboratory is the exothermic reaction which occurs if too much glue is applied. This results in a hard bluish-white coagulum deposited on top of the skin join. In surgery this must be avoided as it burns the skin edges. In postmortem work it should be avoided for aesthetic reasons.

The glue costs $£ 8.00$ an ampoule and is produced in sterile ampoules containing $0.5 \mathrm{~g}$ of the monomer. Each ampoule is sufficient to repair two fetuses once the technique is learnt. As sterility is not a problem in the postmortem room the partially used, already opened ampoules can be re-used. The adhesive also does not polymerise if kept in the refrigerator after opening. As only some fetuses would require repair the cost should not impinge greatly on a laboratory budget.

The adhesive itself is blue. This is to ensure its visibility when applied to living tissue. Unfortunately fetal skin is so delicate that the blue colour sometimes shows through. After discussion with the manufacturers we were sent a sample of colourless glue for laboratory purposes. The disadvantage was that more was used than necessary as it was invisible on the body. This meant more glue, hence more expense, and also a more obvious exothermic response.

The acceptance of the Polkinghorne Report ${ }^{1}$ by the Department of Health and its recommendations means that parents will be able to adopt a more positive attitude towards the post mortem examination of their fetuses. It will become increasingly important for excellent repair of bodies to be the norm. There is no doubt that Histoacryl blue provides an excellent and speedy way to repair fetuses aesthetically.

We thank Dr Farrow of Davis \& Geck for the gift of 25 ampoules of Histoacryl blue.

1 Polkinghorne J. Review of the guidance on the research use of fetuses and fetal materials. Command 762. London: HMSO, 1989. 